\title{
Cardiac magnetic resonance imaging in pulmonary arterial hypertension
}

\author{
Andrew J. Peacock ${ }^{1}$ and Anton Vonk Noordegraaf ${ }^{2}$ \\ Affiliations: 'Scottish Pulmonary Vascular Unit, Glasgow, UK. ${ }^{2}$ Dept of Pulmonology, VU University Medical \\ Center, Amsterdam, The Netherlands.
}

Correspondence: A.J. Peacock, Scottish Pulmonary Vascular Unit, Golden Jubilee National Hospital, Glasgow, G81 4DY, UK. E-mail: apeacockaudcf.gla.ac.uk

ABSTRACT Cardiac magnetic resonance imaging (CMRI) provides accurate information about right ventricular (RV) mass, RV volumes and other markers of RV function. CMRI is proving to be a particularly useful tool in pulmonary arterial hypertension (PAH), as measures of RV function have been shown to be prognostic of long-term outcomes in this disease. Changes in RV function can also provide important information about a patient's disease course and response to treatment. As CMRI is noninvasive it can be used to regularly monitor patients with $\mathrm{PAH}$, which is an important advantage over invasive right heart catheterisation. This review will explore the use of CMRI in the context of existing monitoring tools for $\mathrm{PAH}$ and will explore the forthcoming developments that are likely to be important in the future monitoring of patients with $\mathrm{PAH}$.

0 @ERSpublications

Cardiac MRI provides the gold standard for the measurement of right ventricular shape and function in patients with PAH http://ow.ly/pyIpx

\section{Introduction}

Cardiac magnetic resonance imaging (CMRI) is a noninvasive tool that provides high-resolution, threedimensional images of the heart. It provides information about right heart structure, volumes and function that is not readily obtained via other methods, such as echocardiography and right heart catheterisation (RHC). In pulmonary arterial hypertension (PAH), vasoconstriction and vascular remodelling contribute to a progressive increase in pulmonary vascular resistance (PVR) and pulmonary artery pressure (PAP), which has critical effects on the heart and, in particular, the right ventricle (RV). The ability of the RV to adapt to the increased afterload resulting from rising PVR is the main determinant of a patient's functional capacity and survival. Despite PAH primarily being a disease of the pulmonary vasculature, it is in fact RV failure that is the most common cause of morbidity and death in patients with PAH [1]. Changes to the RV due to pressure overload in PAH are complex, with increased RV wall stress leading to RV dilation and hypertrophy which affect the structure and functioning of the RV as it adapts to increased PAP [2]. Measurements of RV function have been shown to be important in determining patients' prognosis and also, in some cases, response to treatment [3-7]. Given the important implications of RV structure and function to morbidity and mortality in PAH, ongoing and regular assessment is critical.

Guidelines recommend a number of measures to assess disease severity, response to treatment and prognosis in PAH. Many of these measures relate directly to RV function (for example, right atrial pressure and cardiac index) or correlate with measures of RV function (for example, exercise capacity) [8]. While RHC remains the gold standard for PAH diagnosis, there is a need for simple, reproducible, accurate and

Received: Aug 302013 | Accepted after revision: Oct 022013

Conflict of interest: Disclosures can be found alongside the online version of this article at err.ersjournals.com

Provenance: Publication of this peer-reviewed article was supported by Actelion Pharmaceuticals Ltd, Switzerland (principal sponsor, European Respiratory Review issue 130).

Copyright OERS 2013. ERR articles are open access and distributed under the terms of the Creative Commons Attribution Non-Commercial Licence 3.0. 
easy-to-use methods to monitor patients. The purpose of this review is to compare CMRI with the other monitoring techniques currently used for patients with $\mathrm{PAH}$, and to discuss future uses and developments in CMRI techniques.

\section{Current monitoring tools and the "ideal": how does CMRI compare?}

The primary requirements for a monitoring tool for patients with $\mathrm{PAH}$, as in other diseases, are that it should be reproducible and observer independent, standardised, prognostic and sensitive to treatment. Along with this, the ideal tool should also be noninvasive and, where possible, "patient friendly", not timeconsuming and cost-effective. Current guidelines recommend a range of monitoring tools, including clinical markers (e.g. 6-min walk distance (6MWD)), biomarkers (e.g. N-terminal pro-brain natriuretic peptide (NT-proBNP)), invasive measures (e.g. RHC) and noninvasive methods including echocardiography and CMRI [8]. Each has benefits and drawbacks as a monitoring tool.

Change in 6MWD has been used widely as a key end-point in many PAH trials. Its contribution to PAH trials to date, along with its limitations as a primary end-point, are described in more detail by GAINE and Simonneau [9] in this issue of the European Respiratory Review. The 6-min walk test is relatively easy to perform, does not require special equipment and is, therefore, inexpensive. 6MWD does not solely reflect cardiac function and can be affected by factors such as motivation and mood, comorbidities, patient age, height, weight and muscular training $[10,11]$. There is also evidence that the sensitivity of the 6 MWD may decline in line with increasing baseline distance walked, making it potentially less sensitive in patients with less severe disease [12]. A patient's baseline 6MWD has been shown to be prognostic of survival [13]; however, changes in 6MWD do not relate to outcomes in patients with $\mathrm{PAH}$ [14]. In addition, 6MWD has not been fully validated in patients with forms of PAH other than idiopathic PAH (IPAH) [8]. Among potential biomarkers, NT-proBNP, an inactive fragment of a 108 amino acid peptide, is the most studied in $\mathrm{PAH}$. The 108 amino acid peptide is also metabolised to an active fragment BNP; although BNP is less stable than NT-proBNP and, as such, may be perceived as a less convenient marker. Serum levels of NTproBNP have been shown to correlate with mean PAP (mPAP), resting arterial pressure, total pulmonary resistance and RV mass, and they inversely correlate with cardiac output and ejection fraction [15-17]. Although high plasma levels and, in particular, a further increase in plasma BNP, have a strong independent association with mortality $[16,18]$, and there is evidence to support cut-off values associated with survival [17], absolute values cannot be used as an indication of the degree of associated improvement or decline. While RHC remains the gold standard for PAH diagnosis and meets the primary requirements for an ideal monitoring tool, its practicality as a monitoring tool is limited by its invasive nature and the need for it to be performed in centres with the appropriate expertise. High-resolution computed tomography, contrastenhanced computed tomography and pulmonary angiography are particularly useful in assessing chronic thromboembolic pulmonary hypertension (CTEPH). However, although computed tomography can be used to evaluate parameters such as cardiac function and myocardial perfusion, which are relevant to PAH, its use as a monitoring tool is limited by its relatively high radiation exposure.

As noninvasive techniques, both echocardiography and CMRI meet many of the criteria of an ideal monitoring tool for PAH. A range of prognostic factors associated with right heart structure and function have been identified for both echocardiography and CMRI in a number of studies, although many remain to be validated [19]. Overall, echocardiography is a well-established technique that is widely available, inexpensive and safe, and has proven value in the screening and management of patients with PAH. However, it can be associated with a number of technical drawbacks and limitations around operator dependency and problems with obtaining suitable images [20]. Of particular importance to the heart, conventional echocardiography provides two-dimensional images that make the assessment of anatomically complex structures, such as the highly trabeculated RV, difficult. Given that volume calculations of the RV are the basis for quantification assessment of RV systolic function, the inability to measure these parameters accurately by echocardiography is limiting. In addition, it is difficult to evaluate contractile motion and functional parameters within the limitations of two-dimensional echocardiography. In recent years improvements in the image quality have meant that three-dimensional echocardiography has become more widely used and studies have shown that this can be useful in detecting some measures of RV dysfunction [21-25]. However, due to the image quality and level of detail that can be obtained via three-dimensional echocardiography, it is suggested that this technique might be most effective when used in addition to CMRI as standard [25].

CMRI has features that make it particularly suitable for imaging the anatomical complexity of the RV, and is regarded as the gold standard for quantifying ventricular volume, mass, structure and function. It is noninvasive, uses non-toxic contrast agents and does not involve the use of ionising radiation. CMRI provides high-resolution, three-dimensional images that avoid the need for the geometrical assumptions required for some calculations in echocardiography. However, CMRI is also associated with a range of 
limitations: higher cost, more limited availability relative to other methods, the need for more intensive and time-consuming analysis, and the requirement for significant technical support and expertise. In addition, CMRI is incompatible with pacemakers and infusion pumps, and the need for breath holding may be difficult for PAH patients $[19,26]$. However, given the relevance of the right heart in PAH, the advantages of the method may outweigh these disadvantages in the monitoring of patients with established PAH and in assessment of treatment response in clinical trials.

\section{CMRI in PAH}

CMRI is able to provide information on RV mass and changes in mass, RV volumes and changes in volume, RV function and relation to other markers, RV damage, and RV/left ventricle (LV) interaction, meaning it has great potential in PAH. Figure 1 provides examples of CMRI in PAH patients with differing prognosis and disease severity. Although well known for its ability to provide detailed anatomical information, CMRI can also provide functional, perfusion, viability, haemodynamic and metabolic information about the heart and pulmonary system. CMRI measures of ventricular volume and mass have been shown to be reproducible and accurate, and superior to echocardiography [27-29]. Flow velocity can be assessed by encoding the CMRI signal and can be used to calculate phase volumetric flow, which can be further used to determine a range of parameters, including end-diastolic volume, end-systolic volume and stroke volume, cardiac output and ejection fraction. However, although CMRI is an excellent technique for the assessment of volumes and flows, it is less useful for estimating PAP [30, 31]. CMRI has been used to assess systolic PAP [32] and PVR [33] but is not yet considered to be an accurate replacement for measurement performed by RHC.

An increasing number of studies and investigations using CMRI in patients with PAH have been published and identified a range of different variables. Many of these have been shown to be strongly predictive of mortality and survival and, therefore, offer potential for monitoring and assessing response to treatment [19]. Of these, stroke volume is emerging as a key CMRI prognostic measure in PAH patients. Maintenance of normal stroke volume at rest and during exercise in PAH indicates that the RV is well adapted to the increase in afterload placed on it by increasing PVR and PAP. Low stroke volume has been shown to be strongly linked to mortality in IPAH both at baseline and on-treatment (fig. 2) [4, 5], and in PAH related to systemic sclerosis [34]. In patients with PAH and CTEPH, 1 year of treatment with PAH-specific therapy resulted in significant improvements in stroke volume which was related to an increase in 6MWD [4]. Furthermore, the authors were able to calculate a minimal important difference, representing a clinically relevant improvement (in SVI) of $10 \mathrm{~mL}$, which could be used to interpret changes in stroke volume during clinical follow-up or in clinical trials, although validation in a wider range of $\mathrm{PAH}$ aetiologies is required.

Right ventricular ejection fraction (RVEF) is also emerging as an important CMRI-measured prognostic factor in PAH [5, 6]. A study by VAN DE VEERDONK et al. [6] found that RVEF at baseline was a stronger predictor of mortality than PVR. This study also showed that changes in PVR following therapy were only moderately correlated with changes in RVEF, and were not associated with outcome, whereas changes in RVEF were independently related to mortality. Overall, $25 \%$ of patients with decreased PVR following treatment in this study had a decline in RVEF, indicating that RV function does not necessarily adapt to treatment-induced changes in PVR. In patients with a deterioration in RVEF, this was associated with poor
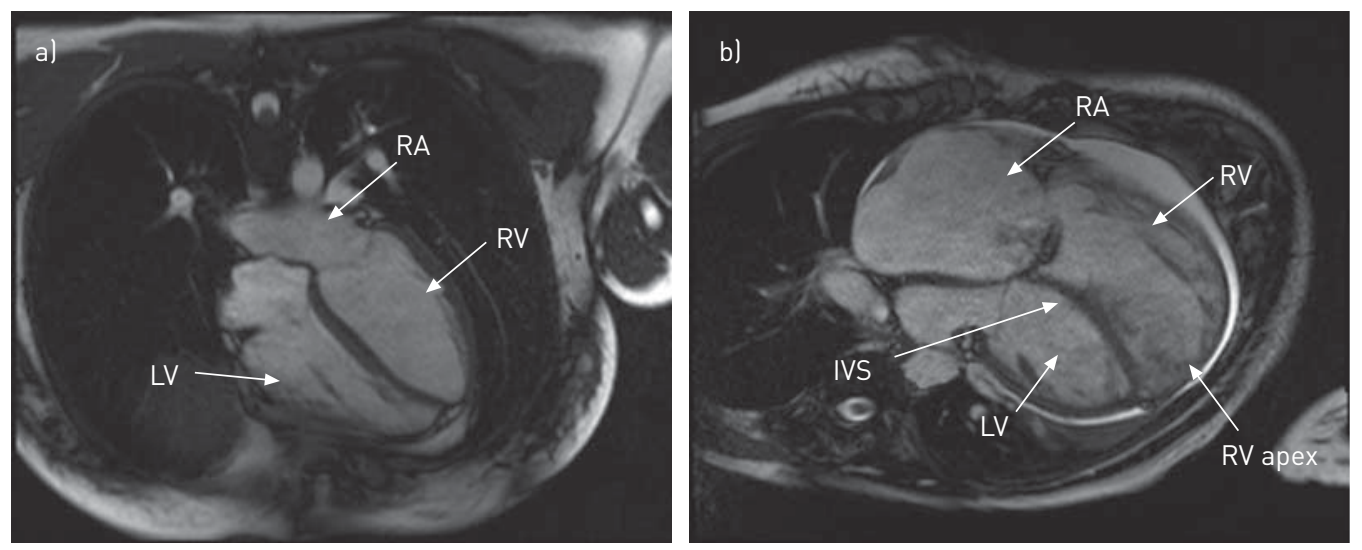

FIGURE 1 Cardiac magnetic resonance imaging in patients with pulmonary hypertension. a) Patient showing right ventricular hypertrophy with normal sized right and left ventricular volumes. b) Patient with end-stage right ventricular failure and a severely dilated right ventricle and atrium. Where the septum is bulging to the left, the right ventricular apex is blunted. RV: right ventricle; LV: left ventricle; RA: right atrium; IVS: interventricular septum. 

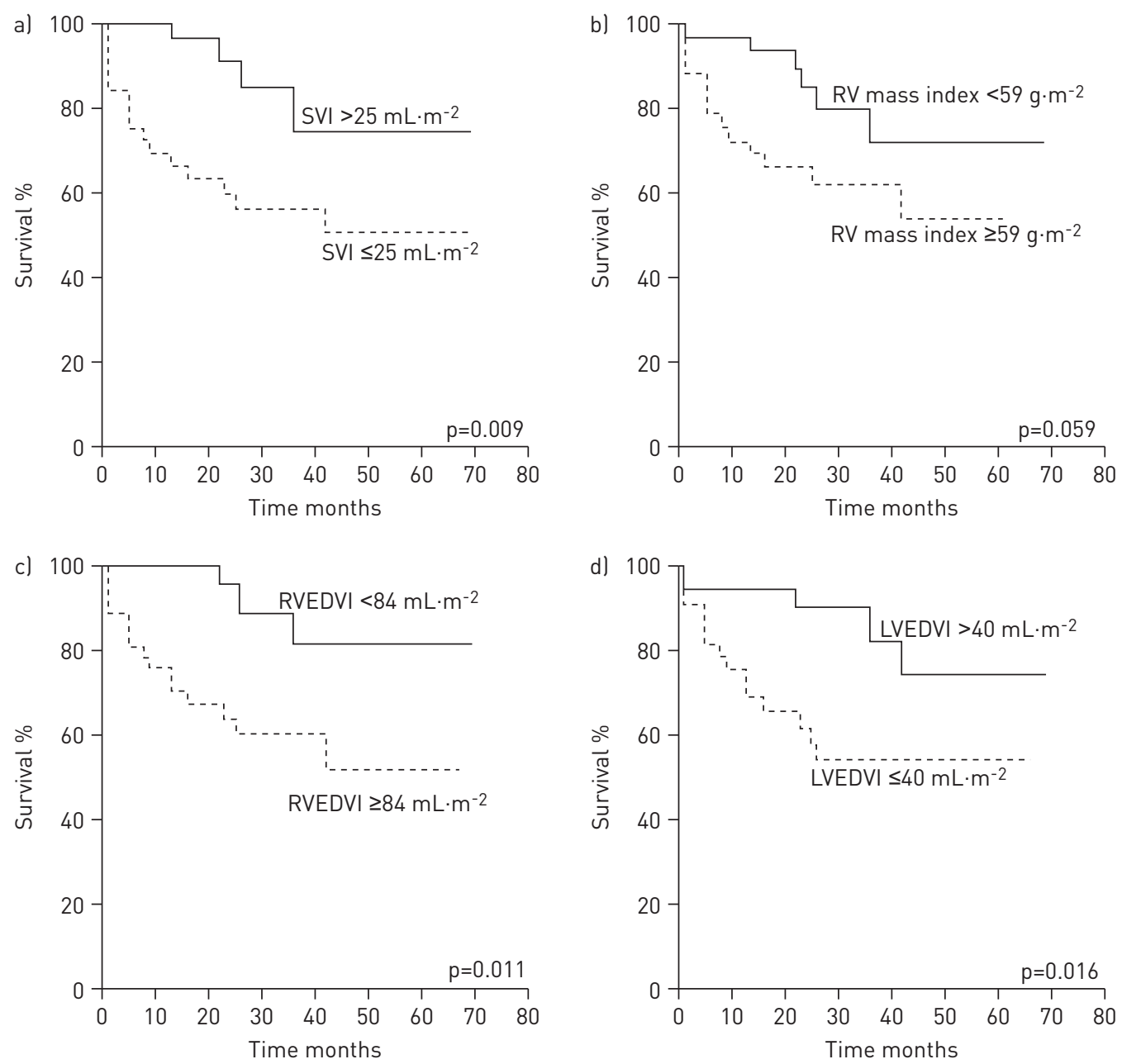

FIGURE 2 Kaplan-Meier survival curves for baseline cardiac magnetic resonance imaging variables according to the mean value in patients with pulmonary hypertension. a) Stroke volume index (SVI), b) right ventricle (RV) mass index, c) right ventricular end-diastolic volume index (RVEDVI), and d) left ventricular end-diastolic volume index (LVEDVI). Reproduced from [5] with permission from the publisher.

outcome, irrespective of improvements in PVR, emphasising the importance of monitoring RV function during the course of the disease (fig. 3) [6].

LV and RV end-diastolic volume are also independent predictors of prognosis in IPAH (fig. 2), and changes in both parameters following treatment are independent predictors of mortality $[5,35]$. A decline in both $\mathrm{LV}$ and RV end-diastolic volume was seen in PAH patients who failed to survive for $>5$ years in a recent study by MAURITZ et al. [36]. This study also compared changes in RV geometric shortening using RV longitudinal and transverse shortening and RV fractional area change, and found that longitudinal shortening, transverse shortening and RV fractional area change, measured at the beginning of the study and 1 year later, were significantly higher in subsequent 5 -year survivors than in nonsurvivors. As transverse shortening incorporates both free-wall and septum movements, the authors suggest that this parameter could be used to monitor the decline in RV function in end-stage PAH [36].

Although a relatively new imaging technique in PAH compared with echocardiography, CMRI is likely to prove a useful monitoring tool for physicians in clinical practice as techniques develop and new markers emerge. For CMRI to become routine, this will require the development of protocols to standardise methodology and to reduce the time taken and the cost associated with CMRI. Validation of the CMRI variables in PAH will also enhance its use in clinical practice. CMRI is also likely to play a key role in determining the potential efficacy of new treatments in PAH. As morbidity and mortality end-points become the new gold standard end-point for clinical trials, large numbers of patients will be required to demonstrate treatment efficacy. Some classical measures, in particular PAP, which are considered to be related to RV dysfunction, are not validated in CMRI. Although some authors have used this finding to 


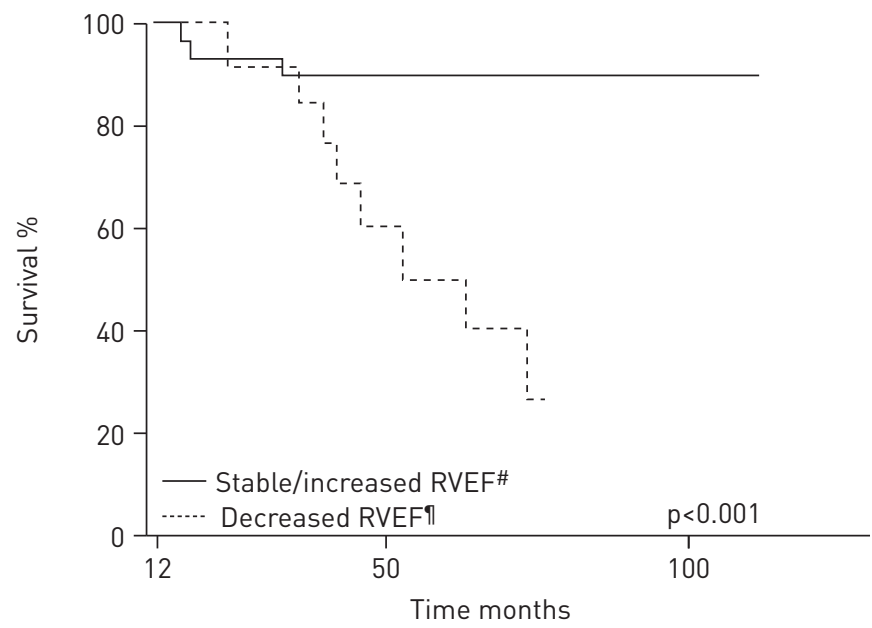

FIGURE 3 Survival of patients with decreased pulmonary vascular resistance at month 12. RVEF: right ventricular ejection fraction. ${ }^{\#}: \mathrm{n}=39 ;{ }^{\uparrow}: \mathrm{n}=13$. Reproduced from [6] with permission from the publisher.

question the validity of CMRI, it has been established in a number of trials that PAP per se is not prognostic in PAH and it is the RV function that determines survival. Therefore, an inability to measure PAP is not a disadvantage as part of a monitoring system. As CMRI can be used to measure relevant and prognostic changes in the heart, it can be used to show whether treatment has a meaningful effect in the short term, and it is likely to be particularly important in phase II studies. Data from the large, prospective, multicentre, longitudinal EURO-MR study, conducted as part of the European Union Framework 6 Pulmotension initiative, will provide the first prospective assessment of the use of CMRI parameters as noninvasive measures of response, and also their correlation with classical measures, including 6MWD, in patients treated with PAH-specific therapy. Data from the study will help confirm the usefulness of CMRI in assessing response to treatment in patients with $\mathrm{PAH}$.

\section{Beyond conventional CMRI}

CMRI techniques can be used to give insight into the function, as well as the structure, of the myocardium. For example, the remodelling and RV myocardial hypertrophy which occur in patients with PAH lead to a need for increased myocardial perfusion. However, increased wall tension leads to decreased oxygen supply, increased oxygen extraction and decreased perfusion secondary to compression of the coronary circulation and impairment of coronary flow [37, 38]. Perfusion CMRI can be used to investigate myocardial perfusion and, thus, gain insights into RV dysfunction in patients with PAH. Using adenosine stress perfusion CMRI imaging, VogeL-CLAUSSEN et al. [37] showed that patients with PAH had significantly reduced biventricular myocardial perfusion reserve which was inversely correlated with RV workload and ejection fraction. Although further studies are needed to determine the prognostic relevance in patients with PAH, these data suggest that reduced myocardial perfusion reserve may contribute to RV dysfunction.

The measurement of ventricular strain, i.e. the motion of the RV wall with contraction, by CMRI can be used to identify regional dysfunction (reduced myocardial contractility) in patients with normal global RV function [39]. Using this technique, an association between reduced biventricular regional function was shown to be associated with increased RV afterload (mPAP and PVR index) [39].

LV early-diastolic strain index, defined as left ventricular free-wall strain in the period between LV free-wall and RV free-wall peak shortening normalised for total systolic LV free-wall shortening, has been shown to be increased in IPAH and CTEPH patients, and to negatively correlate with RVEF and RV stroke volume index [40]. Using computer simulations, LV early-diastolic strain index was shown to reflect inadequate structural adaptation of the RV free wall to increased myofibre load, and may therefore represent a useful, noninvasive predictor of pulmonary hypertension-induced RV dysfunction [40].

A number of studies suggest that contrast-enhanced magnetic resonance imaging (MRI) may be a useful tool in the management of patients with PAH. Injection of a contrast agent, typically gadolinium-based, can produce late gadolinium enhancement (LGE) of cardiac features that have infarct-related scars, or some myocardial fibrosis. LGE is well established as a marker of myocardial abnormalities in a variety of illnesses, including myocardial infarction, fibrosis and inflammation. A number of studies have also reported the presence of LGE in the right ventricular insertion point (RVIP) at the inter-ventricular septum (between the RV and LV) of patients with PAH. Results suggest a significant inverse correlation between the degree of RVIP-LGE and RVEF and haemodynamics [41-44]. Recently, RVIP-LGE has been shown to be associated 
with RV dilation and hypertrophy, reduced RVEF and more extensive haemodynamic abnormalities, as well as a univariate predictor of time to clinical worsening (fig. 4) [45]. As a readily visible and noninvasive marker, RVIP-LGE may be useful as an indicator of more advanced RV changes in PAH and, as a prognostic marker, it may be useful in risk stratification so that appropriate therapy can be administered in a timely manner [45].

The combination of MRI with other imaging techniques may also provide useful information. Positron emission tomography (PET)-MRI combines functional imaging via PET and structural and soft tissue images via MRI within a single imaging session. The biggest technical challenge to be overcome when combining PET and MRI was that the PET detector technology was incompatible with the MRI magnetic field. However, this has been overcome. Although predominantly found in research institutions, PET-MRI devices are slowly becoming available to the wider imaging community. To date, the majority of studies using PET-MRI have been in the oncology and neurology settings; however, there is increasing interest in its use in cardiology [46, 47]. With an ever-increasing range of tracers available, PET-MRI has the potential to provide information that is not available via any other tool, which may enable understanding at the morphological, functional and molecular levels of the complex pathophysiology of PAH and its effects on the heart. This could then potentially show the impact of drugs on the disease process. For example, BOKHARI et al. [48] found that PET scanning using ${ }^{13} \mathrm{~N}_{-} \mathrm{NH}_{3}$ for perfusion imaging and ${ }^{18} \mathrm{~F}$-fluorodeoxyglucose for metabolic imaging was a feasible method for quantifying RV blood flow and metabolism in patients with IPAH. The shift in myocardial glucose uptake seen in this study was suggested to be an early marker of RV dysfunction in the presence of largely preserved RV function on CMRI.
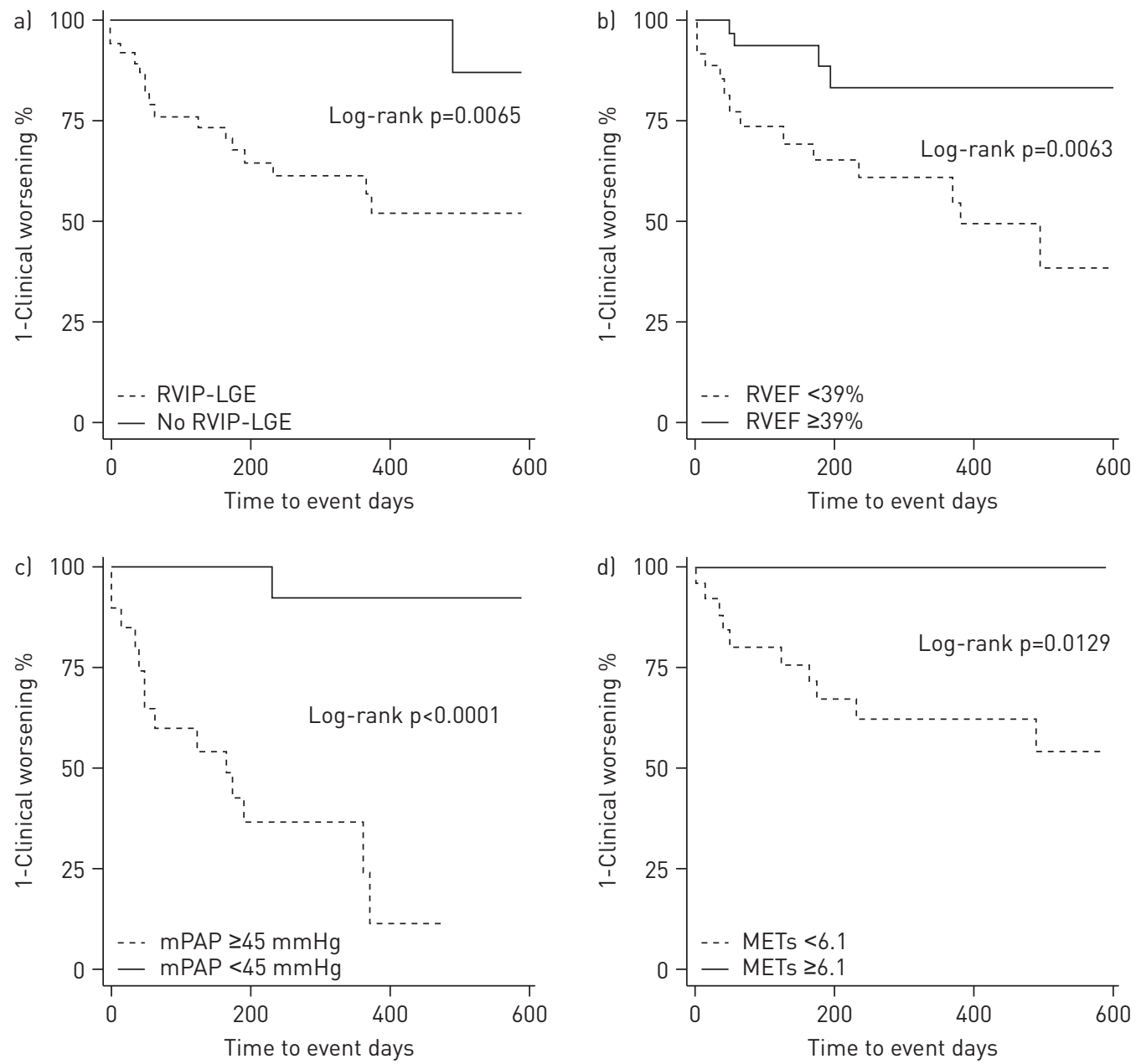

FIGURE 4 Time to clinical worsening for patients with pulmonary hypertension and a) patients with and without the presence of right ventricular insertion point-late gadolinium enhancement (RVIP-LGE), b) patients with right ventricular ejection fraction $(\mathrm{RVEF}) \geqslant 39 \%$ or $<39 \%$, c) patients with mean pulmonary arterial pressure $(\mathrm{mPAP}) \geqslant 45 \mathrm{mmHg}$ or $<45 \mathrm{mmHg}$, and d) patients with metabolic equivalents (METs) $\geqslant 6.1$ and $<6.1$. Reproduced from [45] with permission from the publisher. 
The authors suggest this may be a novel early biomarker that could be a therapeutic target in the treatment and monitoring of PAH.

MRI has the potential to obtain both angiography and perfusion data, offering a comprehensive assessment of vascular morphology and function. In patients with CTEPH, magnetic resonance angiography allows the pulmonary circulation (e.g. webs, vascular obstructions and pulmonary arterial flow) to be evaluated. Given that patients with CTEPH will often require repeated investigations to confirm diagnosis, assess surgical suitability and monitor outcome, magnetic resonance angiography has advantages over conventional and computed tomography pulmonary angiography, which both require the use of radiation and nephrotoxic contrast agents [49]. Time-resolved magnetic resonance angiography allows for both anatomic imaging of the pulmonary vasculature and also evaluation of haemodynamics. Studies have shown that this technique can be used to detect altered haemodynamics in $\mathrm{PAH}$ [50] and variables measured using magnetic resonance angiography are independently associated with MPAP and PVR measured using RHC [51, 52]. MRI has the ability to allow assessment of lung perfusion and, when combined with the measurement of ventricular function and blood flow, is able to provide a unique insight into structure and function in patients with CTEPH.

Although many of these techniques show potential for the assessment of patients with $\mathrm{PAH}$, the prognostic value of the measurements derived by these techniques remains to be validated in larger studies.

\section{Conclusion}

CMRI is already considered the gold standard for assessment of the RV. The increasing appreciation of the key role of the RV in PAH-associated morbidity and mortality means that CMRI, alone or in combination with other techniques, is likely to become increasingly important in the routine assessment and monitoring of PAH and potentially as a measure in clinical trials. Although CMRI has a higher cost, requires expertise and has limited availability, CMRI offers a number of advantages over other tools such as echocardiography. An increasing number of CMRI variables have been shown to be prognostic in PAH patients, and studies have shown that CMRI can be used to detect improvements in cardiac function in response to diseasetargeted therapy. As such, CMRI has great potential as a noninvasive monitoring tool in clinical trials, especially in phase II trials, particularly as the equipment for performing CMRI becomes increasingly available. Future developments including vascular imaging, the use of techniques to assess myocardial damage and conformational changes in the heart, and the combination of CMRI with other imaging techniques have the potential to further extend the usefulness of this technique in the monitoring of patients in the clinic and assessment of treatment response during clinical trials. As these new techniques develop, standardisation and multicentre trials are needed to assess the optimal role of CMRI in patients with PAH.

\section{Acknowledgements}

We would like to thank R. Doyle (Elements Communications Ltd, Westerham, UK) for medical writing support, funded by Actelion Pharmaceuticals Ltd (Allschwil, Switzerland).

\section{References}

Chin KM, Kim NH, Rubin LJ. The right ventricle in pulmonary hypertension. Coron Artery Dis 2005; 16: 13-18. Ho SY, Nihoyannopoulos P. Anatomy, echocardiography, and normal right ventricular dimensions. Heart 2006; 92 : Suppl. 1, i2-i13.

3 D’Alonzo GE, Barst RJ, Ayres SM, et al. Survival in patients with primary pulmonary hypertension. Results from a national prospective registry. Ann Intern Med 1991; 115: 343-349.

4 van Wolferen SA, van de Veerdonk MC, Mauritz GJ, et al. Clinically significant change in stroke volume in pulmonary hypertension. Chest 2011; 139: 1003-1009.

5 van Wolferen SA, Marcus JT, Boonstra A, et al. Prognostic value of right ventricular mass, volume, and function in idiopathic pulmonary arterial hypertension. Eur Heart J 2007; 28: 1250-1257.

6 van de Veerdonk MC, Kind T, Marcus JT, et al. Progressive right ventricular dysfunction in patients with pulmonary arterial hypertension responding to therapy. J Am Coll Cardiol 2011; 58: 2511-2519.

7 Forfia PR, Fisher MR, Mathai SC, et al. Tricuspid annular displacement predicts survival in pulmonary hypertension. Am J Respir Crit Care Med 2006; 174: 1034-1041.

8 Galiè N, Hoeper MM, Humbert M, et al. Guidelines for the diagnosis and treatment of pulmonary hypertension: the Task Force for the Diagnosis and Treatment of Pulmonary Hypertension of the European Society of Cardiology (ESC) and the European Respiratory Society (ERS), endorsed by the International Society of Heart and Lung Transplantation (ISHLT). Eur Heart J 2009; 30: 2493-2537.

9 Gaine S, Simonneau G. The need to move from 6-minute walk distance to outcome trials in pulmonary arterial hypertension. Eur Respir Rev 2013; 22: 487-494.

10 Casanova C, Celli BR, Barria P, et al. The 6-min walk distance in healthy subjects: reference standards from seven countries. Eur Respir J 2011; 37: 150-156.

11 Olsson LG, Swedberg K, Clark AL, et al. Six minute corridor walk test as an outcome measure for the assessment of treatment in randomized, blinded intervention trials of chronic heart failure: a systematic review. Eur Heart J 2005; 26: $778-793$. 
12 Frost AE, Langleben D, Oudiz R, et al. The 6-min walk test (6MW) as an efficacy endpoint in pulmonary arterial hypertension clinical trials: demonstration of a ceiling effect. Vascul Pharmacol 2005; 43: 36-39.

13 Humbert M, Sitbon O, Chaouat A, et al. Survival in patients with idiopathic, familial, and anorexigen-associated pulmonary arterial hypertension in the modern management era. Circulation 2010; 122: 156-163.

14 Savarese G, Paolillo S, Costanzo P, et al. Do changes of 6-minute walk distance predict clinical events in patients with pulmonary arterial hypertension? A meta-analysis of 22 randomized trials. J Am Coll Cardiol 2012; 60: 1192-1201.

15 Nagaya N, Nishikimi T, Okano Y, et al. Plasma brain natriuretic peptide levels increase in proportion to the extent of right ventricular dysfunction in pulmonary hypertension. J Am Coll Cardiol 1998; 31: 202-208.

16 Fijalkowska A, Kurzyna M, Torbicki A, et al. Serum N-terminal brain natriuretic peptide as a prognostic parameter in patients with pulmonary hypertension. Chest 2006; 129: 1313-1321.

17 Blyth KG, Groenning BA, Mark PB, et al. NT-proBNP can be used to detect right ventricular systolic dysfunction in pulmonary hypertension. Eur Respir J 2007; 29: 737-744.

18 Nagaya N, Nishikimi T, Uematsu M, et al. Plasma brain natriuretic peptide as a prognostic indicator in patients with primary pulmonary hypertension. Circulation 2000; 102: 865-870.

19 Vonk Noordegraaf A, Galie N. The role of the right ventricle in pulmonary arterial hypertension. Eur Respir Rev 2011; 20: 243-253.

20 Badano LP, Ginghina C, Easaw J, et al. Right ventricle in pulmonary arterial hypertension: haemodynamics, structural changes, imaging, and proposal of a study protocol aimed to assess remodelling and treatment effects. Eur J Echocardiogr 2010; 11: 27-37.

21 Grapsa J, O’Regan DP, Pavlopoulos H, et al. Right ventricular remodelling in pulmonary arterial hypertension with three-dimensional echocardiography: comparison with cardiac magnetic resonance imaging. Eur J Echocardiogr 2010; 11: 64-73.

22 Grapsa J, Gibbs JS, Dawson D, et al. Morphologic and functional remodeling of the right ventricle in pulmonary hypertension by real time three dimensional echocardiography. Am J Cardiol 2012; 109: 906-913.

23 Grapsa J, Gibbs JS, Cabrita IZ, et al. The association of clinical outcome with right atrial and ventricular remodelling in patients with pulmonary arterial hypertension: study with real-time three-dimensional echocardiography. Eur Heart J Cardiovasc Imaging 2012; 13: 666-672.

24 Kong D, Shu X, Pan C, et al. Evaluation of right ventricular regional volume and systolic function in patients with pulmonary arterial hypertension using three-dimensional echocardiography. Echocardiography 2012; 29: 706-712.

25 Bhave NM, Patel AR, Weinert L, et al. Three-dimensional modeling of the right ventricle from two-dimensional transthoracic echocardiographic images: utility of knowledge-based reconstruction in pulmonary arterial hypertension. J Am Soc Echocardiogr 2013; 26: 860-867.

26 Benza R, Biederman R, Murali S, et al. Role of cardiac magnetic resonance imaging in the management of patients with pulmonary arterial hypertension. J Am Coll Cardiol 2008; 52: 1683-1692.

27 Bottini PB, Carr AA, Prisant LM, et al. Magnetic resonance imaging compared to echocardiography to assess left ventricular mass in the hypertensive patient. Am J Hypertens 1995; 8: 221-228.

28 Grothues F, Smith GC, Moon JC, et al. Comparison of interstudy reproducibility of cardiovascular magnetic resonance with two-dimensional echocardiography in normal subjects and in patients with heart failure or left ventricular hypertrophy. Am J Cardiol 2002; 90: 29-34.

29 Grothues F, Moon JC, Bellenger NG, et al. Interstudy reproducibility of right ventricular volumes, function, and mass with cardiovascular magnetic resonance. Am Heart J 2004; 147: 218-223.

30 Saba TS, Foster J, Cockburn M, et al. Ventricular mass index using magnetic resonance imaging accurately estimates pulmonary artery pressure. Eur Respir J 2002; 20: 1519-1524.

31 Laffon E, Vallet C, Bernard V, et al. A computed method for noninvasive MRI assessment of pulmonary arterial hypertension. J Appl Physiol 2004; 96: 463-468.

32 Nogami M, Ohno Y, Koyama H, et al. Utility of phase contrast MR imaging for assessment of pulmonary flow and pressure estimation in patients with pulmonary hypertension: comparison with right heart catheterization and echocardiography. J Magn Reson Imaging 2009; 30: 973-980.

33 García-Alvarez A, Fernández-Friera L, Mirelis JG, et al. Non-invasive estimation of pulmonary vascular resistance with cardiac magnetic resonance. Eur Heart J 2011; 32: 2438-2445.

34 Campo A, Mathai SC, Le Pavec J, et al. Hemodynamic predictors of survival in scleroderma-related pulmonary arterial hypertension. Am J Respir Crit Care Med 2010; 182: 252-260.

35 Yamada Y, Okuda S, Kataoka M, et al. Prognostic value of cardiac magnetic resonance imaging for idiopathic pulmonary arterial hypertension before initiating intravenous prostacyclin therapy. Circ J 2012; 76: 1737-1743.

36 Mauritz GJ, Kind T, Marcus JT, et al. Progressive changes in right ventricular geometric shortening and long-term survival in pulmonary arterial hypertension. Chest 2012; 141: 935-943.

37 Vogel-Claussen J, Skrok J, Shehata ML, et al. Right and left ventricular myocardial perfusion reserves correlate with right ventricular function and pulmonary hemodynamics in patients with pulmonary arterial hypertension. Radiology 2011; 258: 119-127.

38 van Wolferen SA, Marcus JT, Westerhof $\mathrm{N}$, et al. Right coronary artery flow impairment in patients with pulmonary hypertension. Eur Heart J 2008; 29: 120-127.

39 Shehata ML, Harouni AA, Skrok J, et al. Regional and global biventricular function in pulmonary arterial hypertension: a cardiac MR imaging study. Radiology 2013; 266: 114-122.

40 Lumens J, Arts T, Marcus JT, et al. Early-diastolic left ventricular lengthening implies pulmonary hypertensioninduced right ventricular decompensation. Cardiovasc Res 2012; 96: 286-295.

41 Blyth KG, Groenning BA, Martin TN, et al. Contrast enhanced-cardiovascular magnetic resonance imaging in patients with pulmonary hypertension. Eur Heart J 2005; 26: 1993-1999.

42 McCann GP, Gan CT, Beek AM, et al. Extent of MRI delayed enhancement of myocardial mass is related to right ventricular dysfunction in pulmonary artery hypertension. AJR Am J Roentgenol 2007; 188: 349-355.

43 Sanz J, Dellegrottaglie S, Kariisa M, et al. Prevalence and correlates of septal delayed contrast enhancement in patients with pulmonary hypertension. Am J Cardiol 2007; 100: 731-735.

44 Junqueira FP, Macedo R, Coutinho AC, et al. Myocardial delayed enhancement in patients with pulmonary hypertension and right ventricular failure: evaluation by cardiac MRI. Br J Radiol 2009; 82: 821-826. 
Freed BH, Gomberg-Maitland M, Chandra S, et al. Late gadolinium enhancement cardiovascular magnetic resonance predicts clinical worsening in patients with pulmonary hypertension. J Cardiovasc Magn Reson 2012; 14: 11.

46 Anagnostopoulos C, Georgakopoulos A, Pianou N, et al. Assessment of myocardial perfusion and viability by positron emission tomography. Int J Cardiol 2013; 167: 1737-1749.

47 Nekolla SG, Martinez-Moeller A, Saraste A. PET and MRI in cardiac imaging: from validation studies to integrated applications. Eur J Nucl Med Mol Imaging 2009; 36: Suppl. 1, S121-S130

48 Bokhari S, Raina A, Rosenweig EB, et al. PET imaging may provide a novel biomarker and understanding of right ventricular dysfunction in patients with idiopathic pulmonary arterial hypertension. Circ Cardiovasc Imaging 2011; 4: 641-647.

49 McNeil K, Dunning J. Chronic thromboembolic pulmonary hypertension (CTEPH). Heart 2007; 93: 1152-1158.

50 Jeong HJ, Vakil P, Sheehan JJ, et al. Time-resolved magnetic resonance angiography: evaluation of intrapulmonary circulation parameters in pulmonary arterial hypertension. J Magn Reson Imaging 2011; 33: 225-231.

51 Swift AJ, Rajaram S, Condliffe R, et al. Pulmonary artery relative area change detects mild elevations in pulmonary vascular resistance and predicts adverse outcome in pulmonary hypertension. Invest Radiol 2012; 47: 571-577.

52 Sergiacomi G, Bolacchi F, Cadioli M, et al. Combined pulmonary fibrosis and emphysema: 3D time-resolved MR angiographic evaluation of pulmonary arterial mean transit time and time to peak enhancement. Radiology 2010; 254: 601-608. 